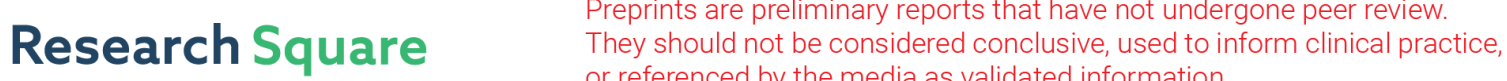 or referenced by the media as validated information. \\ Clear evidence against superconductivity in hydrides under high pressure
}

\author{
J. E. Hirsch ( $\nabla$ jhirsch@ucsd.edu ) \\ University of California, San Diego \\ F. Marsiglio \\ University of Alberta
}

\section{Research Article}

Keywords: hydride superconductivity, magnetization, Meissner effect, lower critical field, trapped flux

Posted Date: February 23rd, 2022

DOI: https://doi.org/10.21203/rs.3.rs-1001084/v3

License: (c) (i) This work is licensed under a Creative Commons Attribution 4.0 International License. Read Full License 


\title{
Clear evidence against superconductivity in hydrides under high pressure
}

\author{
J. E. Hirsch ${ }^{a *}$ and F. Marsiglio ${ }^{b}$ \\ ${ }^{a}$ Department of Physics, University of California, San Diego, La Jolla, CA 92093-0319 \\ ${ }^{*}$ Corresponding author. Email: jhirsch@ucsd.edu \\ ${ }^{b}$ Department of Physics, University of Alberta, Edmonton, Alberta, Canada T6G 2E1
}

\begin{abstract}
The Meissner effect, magnetic field expulsion, is a hallmark of superconductivity. Associated with it, superconductors exclude applied magnetic fields. Recently Minkov et al. presented experimental results reportedly showing "definitive evidence of the Meissner effect" in sulfur hydride and lanthanum hydride under high pressure [1], and more recently Eremets et al. argued that "the arguments against superconductivity (in hydrides) can be either refuted or explained" [2]. Instead, we show here that the evidence presented in those papers does not support the case for superconductivity in these materials. Together with experimental evidence discussed in earlier papers, we argue that this strongly suggests that hydrides under pressure are not high temperature superconductors.
\end{abstract}

PACS numbers:

\section{INTRODUCTION}

The era of high temperature superconductivity in hydrides under high pressure was spawned by the reported discovery of superconductivity in sulfur hydride in 2015 by Eremets and coworkers [3, 4], with critical temperature $203 \mathrm{~K}$, higher than any other critical temperature known before. Since then, it has been reported that superconductivity at high temperatures occurs also in 11 other hydrides under pressure [5-7]. These experimental works are strongly motivated and guided by theoretical predictions of superconductivity in these materials based on the conventional BCS-electron-phonon theory of superconductivity [8-11].

Instead, we have recently argued that the experimental evidence presented so far does not provide conclusive proof of superconductivity in any of these materials [1223]. Others have also questioned experimental [24-26] and theoretical $[27,28]$ evidence for superconductivity in some of these materials. Therefore, the very existence of high temperature superconductivity in hydrides under pressure is now in doubt.

In the initial paper [3], Eremets and coworkers presented magnetic evidence of superconductivity in sulfur hydride, that was questioned in ref. [15]. Recently, Minkov and coworkers provided new magnetic evidence for superconductivity in sulfur hydride as well as in lanthanum hydride [1], and argued that it provides definitive evidence for superconductivity. Eremets et al. provided further arguments and experimental results in ref. [2]. Instead, we argue in this paper that these new measurements together with the old measurements provide convincing evidence against the existence of superconductivity in these hydrides, in our opinion.

\section{COMPARISON OF OLD AND NEW MAGNETIC EVIDENCE FOR SUPERCONDUCTIVITY IN SULFUR HYDRIDE}

To have confidence that experimental results reflect true physics of the material being studied, it is essential that measurements are reproducible, not only within one lab and experimental group but also in different settings. Unfortunately, no other group has reported measurements of magnetization in sulfur hydride (nor any other hydride) under high pressure.

The experimental results on sulfur hydride reported by the Eremets group in 2015 [3] and 2021 [1] report critical temperatures of $203 \mathrm{~K}$ and $196 \mathrm{~K}$ respectively, i.e. they are very close to one another. The size of the samples used is also reported to be similar, approximately $80 \mu \mathrm{m}$ [3] and $85 \mu m$ [1] in diameter and "a few $\mu m$ " in thickness in [3] and between $2.1 \mu \mathrm{m}$ and $3.1 \mu \mathrm{m}$ in thickness in [1]. However, the measured magnetic moment of the samples under an applied magnetic field of the same magnitude differ by a factor of 5, with the 2015 sample having the larger magnetic moment. No explanation for this large discrepancy is given in [1]. These magnetic moments are measured not by field cooling but by applying a magnetic field to an already cold sample, and for sufficiently small fields so that the field should not penetrate the sample, hence such large differences are not expected for samples of similar sizes.

More importantly, the magnitude of the lower critical field reported in 2015 [3] was $30 \mathrm{mT}$, and the same quantity in 2021 is $1.9 \mathrm{~T}$ [1]. That is a difference of a factor of 60 . The magnitude of the London penetration depth was reported to be $125 \mathrm{~nm}$ in 2015 [3] and $12.7 \mathrm{~nm}$ in 2021 [1], a difference of a factor of 10.

Thus, for the past 6 years the physics community was asked to believe that sulfur hydride had been proven to be a $203 \mathrm{~K}$ superconductor based on magnetic measurements that were wrong by these very significant factors. The magnetic evidence reported by Eremets and coworkers in 2015 [3] was regarded by many to be the strongest evidence that hydrides under pressure are high temper- 

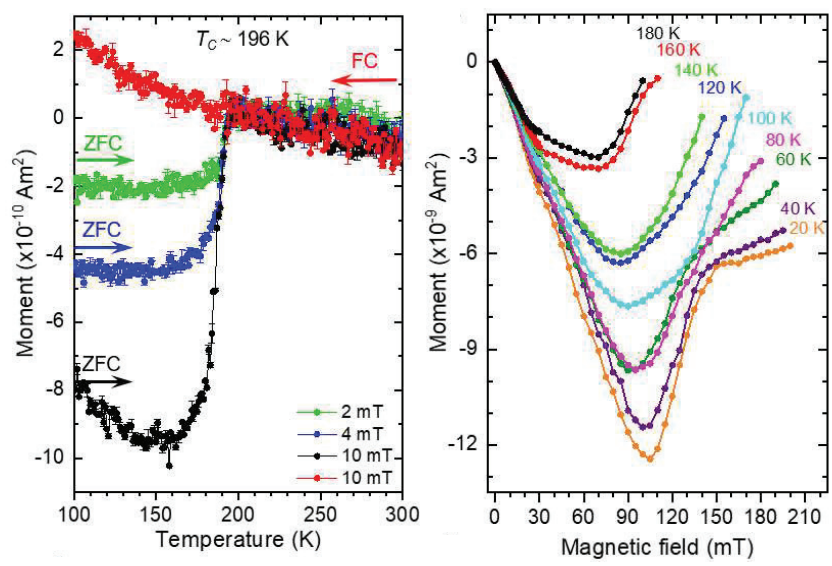

FIG. 1: Left panel: magnetization versus temperature of sulfur hydride reported in ref. [1], under field cooling (FC) and zero field cooling (ZFC). Right panel: magnetization versus applied magnetic field for sulfur hydride reported in ref. [1].

ature superconductors. Now, Eremets et al. themselves are telling us that that evidence was flawed [1]. In other words, the entire edifice of high temperature superconductivity in pressurized hydrides was built on deeply faulty foundations.

\section{MAGNETIC EVIDENCE IN 2021}

It is also remarkable that after the reported magnetization measurements in 2015 using a specially designed miniature nonmagnetic DAC cell that could accomodate a SQUID magnetometer [3], no new experimental results using that sophisticated apparatus and technique were reported for a full 6 years, neither by the authors of ref. [3] nor by anybody else. Yet during those years, about 30 new reports of high temperature superconductivity in 12 different pressurized hydrides were published [5-7].

Fast forward to 2021 [1]. Figure 1 shows on the left panel the reported magnetization versus temperature under an applied magnetic field, and on the right panel the magnetization versus magnetic field for various values of the temperature [1]. It can be seen that no evidence of a superconducting transition is seen under field cooling on the left panel. While this behavior has been observed in some strongly type II superconductors [29, 30], it has never been observed for type I or weakly type II superconductors, to our knowledge. According to ref. [1], this material is a weakly type II superconductor, with Ginzburg-Landau parameter $\kappa=6.9$. The reported London penetration depth is remarkably small, $12.7 \mathrm{~nm}$, indicating that the material has a large superfluid density and small degree of disorder. Such materials always exhibit a robust Meissner effect, i.e. magnetic flux expulsion. We argue that the fact that this material does not show any evidence of magnetic field expulsion under field cooling, the signature of superconductivity, is clear and

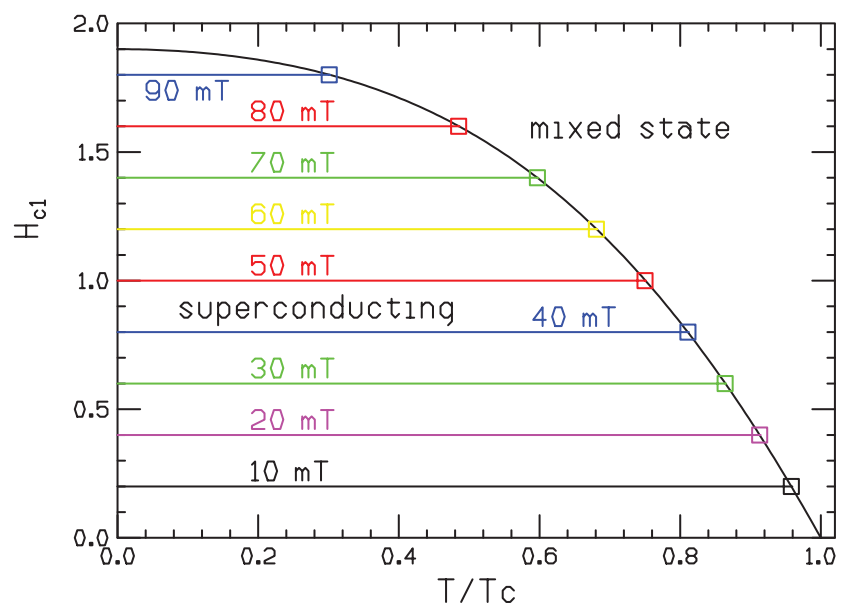

FIG. 2: Lower critical field versus temperature for a standard superconductor according to Ginzburg-Landau theory. The zero temperature critical field is $1.9 \mathrm{~T}$. The numerical values of the field given in $\mathrm{mT}$ indicate the applied field $H_{p}$, that becomes $H_{c 1}=20 H_{p}$ due to the demagnetization factor $1 /(1-N)$ for $N=0.95$ reported in ref. [1].

direct evidence that the material is not a superconductor.

Ref. [1] claims that the sample is a flat disk of demagnetization factor $N=0.95$. From this information, and from the observation that a magnetic field at low temperatures of magnitude $H_{p} \sim 96 m T$ starts to penetrate the sample, as seen in the lowest curve on the right panel of fig. 1, labeled $20 \mathrm{~K}$, the authors infer that the lower critical field of sulfur hydride is $H_{c 1}(T=0)=1.9 T=H_{p} /(1-N)$. In figure 2 we plot the behavior of the lower critical field of a standard superconductor as a function of temperature inferred from Ginzburg-Landau theory. The horizontal lines indicate the values of the lower critical field $H_{c 1}$ for the values of applied field indicated, $H_{p}=10 \mathrm{mT}, 20 \mathrm{mT}$, etc. The squares indicate the critical points for each value of the applied magnetic field.

For each value of the magnetic field, for temperatures lower than that critical value, the magnetic field should be completely excluded from the sample, except for the small region within $\lambda_{L}$ of the surface. Given that the penetration depth is $12.7 \mathrm{~nm}$ and the sample diameter and height are $85 \mu \mathrm{m}$ and $2.8 \mu \mathrm{m}$ respectively, this implies that $99.5 \%$ of the sample remains field free except very close to the critical temperature. Therefore, we expect the magnetization versus temperature to be essentially flat below each critical temperature. This is approximately consistent with what is seen for the three curves shown in the left panel of Fig. 1. For those values of the applied field, $H_{p}=2 m T, 4 m T$ and $10 m T$, the critical temperatures are $194.4 \mathrm{~K}, 192.8 \mathrm{~K}$ and $187.8 \mathrm{~K}$, all close to the zero field critical temperature $196 \mathrm{~K}$.

From the reported values of magnetic moment versus magnetic field shown in the right panel of fig. 1 we can extract the behavior of magnetic moment versus temperature for applied fields larger than the values shown on 


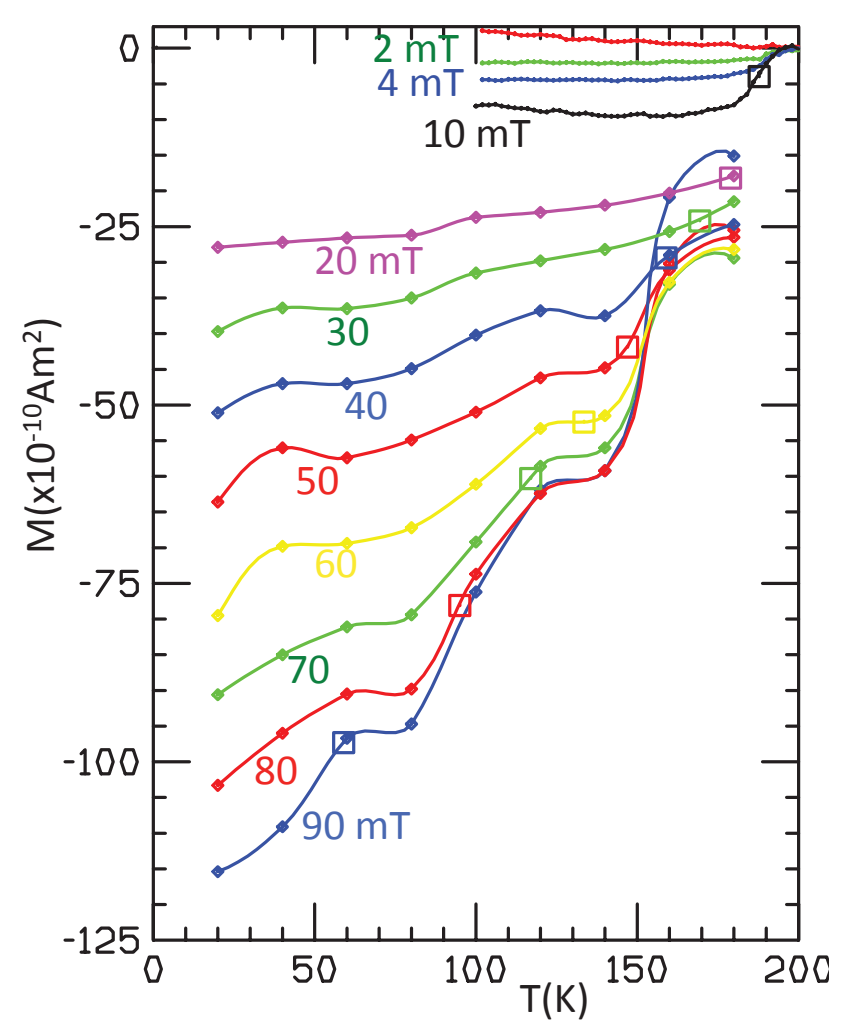

FIG. 3: Magnetic moment versus temperature inferred from the experimental results of ref. [1] shown in Fig. 1, for various values of the applied magnetic field $H_{p}$ given in $m T$ in the figure. The colored squares indicate the point on the curve for the applied magnetic field that corresponds to the critical temperature given by the phase boundary in Fig. 2 .

the left panel of fig. 1. This is shown in fig. 3. The value of the abscissa for each square is the critical temperature for each value of the applied field inferred from fig. 2, and we would expect the magnetic moment to be approximately constant for temperatures lower than those values, since the magnetic field is completely excluded from the sample. The behavior seen in fig. 3 is qualitatively different from this expectation.

Indeed, it can be seen in fig. 3 that the sample is unaware of the fact that it underwent a superconducting transition at its critical temperature, denoted by the square on each curve. The magnetization in each case (except for the selected ones that the authors of ref. [1] chose to show us on the left panel of fig. 1, also reproduced in fig. 3), continues its downward trend as the temperature is lowered further, oblivious to the fact that the magnetic field is no longer in its interior. This could be interpreted as revealing yet another new property of nonstandard superconductors [13] not shared by standard conventional or unconventional superconductors: an ability to change the magnetic field in their surroundings while keeping the magnetic field in their interior constant (i.e. equal to zero).

\section{MEISSNER EFFECT ON STEROIDS AND ENORMOUS DENSITY OF STATES}

In ref. [14], in connection with an analysis of a nuclear resonant scattering experiment on sulfur hydride [31], we pointed out that a lower critical field $H_{c 1} \sim 2.6 T$, a thermodynamic critical field $H_{c} \sim 10.6 T$, and a London penetration depth $\lambda_{L}=10.0 \mathrm{~nm}$ are completely incompatible with the physics of standard superconductors. Those values are remarkably close to the values $H_{c 1}=1.9 \mathrm{~T}$, $H_{c}=9.8 \mathrm{~T}$ and $\lambda_{L}=12.7 \mathrm{~nm}$ that Minkov et al now postulate [1] are the appropriate values for sulfur hydride.

To consider as an alternative a less extreme set of parameters let us assume that due to uncertainty in the dimensions of the sample the demagnetizing factor is $N=0.8$ instead of the $N=0.95$ assumed by Minkov et al. This then yields $H_{c 1}=0.48 \mathrm{~T}, H_{c}=4.1 \mathrm{~T}$ and $\lambda_{L}=20.4 \mathrm{~nm}$ as an alternative to the values assumed by Minkov et al.

Let us start by computing the critical current, assuming the Minkov et al. values. A magnetic field of magnitude smaller than $H_{c 1}=1.9 \mathrm{~T}$, the value for $H_{c 1}$ estimated for sulfur hydride in ref. [1], should be completely excluded from the interior of a long cylinder. The current density circulating near the surface is, according to London's equation

$$
J_{c}=\frac{c}{4 \pi \lambda_{L}} H_{c 1}
$$

which for the above given values of $H_{c 1}$ and $\lambda_{L}$ yields

$$
J_{c}=1.19 \times 10^{10} \mathrm{Amp} / \mathrm{cm}^{2} .
$$

This value is at least two to three orders of magnitude larger than critical currents of any other known superconductor, whether type I or type II, hard or soft [32]. If instead we use our alternative values for the parameters assuming $N=0.8$ given above, the critical current is $J_{c}=1.23 \times 10^{9} \mathrm{Amp} / \mathrm{cm}^{2}$, still much larger than for any other known superconductor.

Instead, ref. [1] estimated a value of the critical current density

$$
J_{c} \sim 7 \times 10^{6} \mathrm{Amp} / \mathrm{cm}^{2}
$$

using magnetization measurements and the Bean model. However, such an estimate can only be valid for a strongly type II superconductor with much smaller $H_{c 1}$ and larger $\lambda_{L}$, such that the value of magnetic field given by the expression $H=\left(4 \pi \lambda_{L} / c\right) J_{c}$ is larger than $H_{c 1}$. In such a case the magnetic field penetrates the sample and generates vortices that are pinned near the sample surface, and the strength of the pinning potential determines the critical current according to the Bean model. That calculation is not applicable for the case at hand here to determine the critical current, since $H=\left(4 \pi \lambda_{L} / c\right) J_{c}$, with $J_{c}$ given by Eq. (3), is much smaller than $H_{c 1}$. 
The density of states at the Fermi energy $g\left(\epsilon_{F}\right)$ can be obtained from the standard theromodynamic relation

$$
\frac{H_{c}^{2}(0)}{8 \pi}=\frac{1}{2} g\left(\epsilon_{F}\right) \Delta^{2}
$$

with $\Delta$ the energy gap at zero temperature. From the standard BCS relation $2 \Delta / k_{B} T_{c}=3.53$ and $T_{c}=196 \mathrm{~K}$ [1] we have $\Delta=29.8 \mathrm{meV}$, and using $H_{c}=9.8 \mathrm{~T}$ yields

$$
g\left(\epsilon_{F}\right)=\frac{0.537 \text { states }}{\text { spin }-e V A^{3}} .
$$

This is an enormous density of states. For comparison, using density functional theory the density of states of sulfur hydride was estimated to be 0.019 states/(spin-eV $\AA^{3}$ ) [10], twenty eight times smaller. Our alternative values for the parameters assuming $N=0.8$ yield a density of states that is smaller than Eq. (5) by a factor of six, so more than four times larger than the expected value.

In ref. [14] we discussed in more detail why such numbers are completely incompatible with standard superconductivity. Barring a qualitatively different superconducting state unlike that of all known superconductors, this implies, in our opinion, that the properties measured in ref. [1] interpreted to "unambiguously confirm superconductivity" in fact indicate that the material that was measured was not a superconductor.

The analysis discussed above for sulfur hydride applies equally well to the measurements for $L a H_{10}$ reported in ref. [1]. It is claimed that for $L a H_{10}$ the zero temperature lower critical field, thermodynamic critical field and London penetration depth are $H_{c 1}=1 \mathrm{~T}, H_{c}=7.4 \mathrm{~T}$ and $\lambda_{L}=20.7 \mathrm{~nm}$ [1], comparable to the values reported for $\mathrm{H}_{3} \mathrm{~S}$ and equally impossible. The resulting critical current density for $\mathrm{LaH}_{10}$ is $3.84 \times 10^{9} \mathrm{Amp} / \mathrm{cm}^{2}$, and the resulting density of states is $g\left(\epsilon_{F}\right)=0.22$ states/spin-eV$\AA^{3}$, much larger than the theoretically estimated value of 0.016 states/spin-eV- $\AA^{3}$ [10]. The magnetic moment behavior reported for $L a H_{10}$ has the same unphysical behavior as shown in the previous section for $H_{3} S$.

\section{SAMPLE QUALITY}

The samples were prepared by pulsed laser heating of a precursor containing $\mathrm{NH}_{3} \mathrm{BH}_{3}$ as a source of hydrogen and either $S$ or $\mathrm{LaH}_{3}$. Then the precursor was heated by traversing a $5 \mu \mathrm{m}$ laser spot horizontally and vertically across the sample.

The fact that the laser spot is much smaller than the estimated diameters of the samples suggests that the resulting sample cannot possibly be a single crystal. Instead, there are likely to be many different regions of the sample of size of order of the laser spot that are connected by weak links (V. Struzhkin, private communication (2021)) that would also give rise to pinning centers that could explain the absence of magnetic flux expulsion upon cooling.
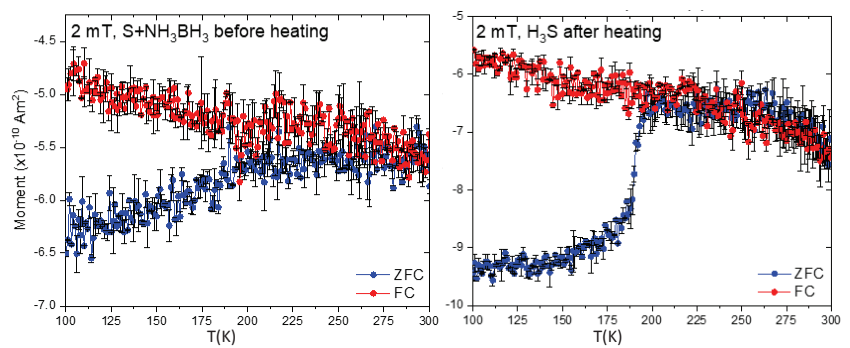

FIG. 4: Lower left (left panel) and middle left (right panel) panels of Fig. SI1 of ref. [1]. The left panel corresponds to the precursor sample, the right panel to the assumed superconducting sample. See text for discussion.

\section{A SMOKING GUN?}

Adding to the arguments presented in the previous sections, we argue that Fig. SI1 of the paper [1] is a smoking gun that provides clear evidence for the faulty analysis and conclusions of ref. [1].

The lower left and middle left panels of Fig. SI1 are reproduced in Fig. 4. The left panel shows the measured magnetic moment versus temperature under an applied magnetic field of $2 \mathrm{mT}$ for the sample precursors, namely $\mathrm{S}+\mathrm{NH}_{3} \mathrm{BH}_{3}$ before undergoing the laser heating process that generates the supposedly superconducting sample. The zero field cooling and field cooling curves approximately coincide for temperatures above $200 \mathrm{~K}$ and diverge below 200K, with the ZFC magnetic moment decreasing and the FC moment increasing as the temperature is further lowered. Precisely the same behavior, attributed to superconductivity, is observed in the sample after heating, shown in the right panel of Fig. 4 (and in the left panel of Fig. 1, red and green curves).

How does the precursor sample know that the critical temperature of the superconducting sample will be close to $200 K$ ? Why does an applied magnetic field give rise to a different magnetization for the non-superconducting sample under field cooling and zero field cooling, also seen in the lower middle and right panels of Fig. SI1 for other values of the applied magnetic field?

The authors attribute the difference in the FC and ZFC curves shown in fig. SI1 to "contamination by magnetic pieces" (ref. [1] and V. Minkov, private communication). Clearly such effects, which will not necessarily have the same temperature dependence before and after heating, and hence cannot be simply subtracted out, could also be responsible for the signals interpreted by the authors as superconductivity.

We argue that the observed behavior in the lower panels of Fig. SI1, which obviously is not indicative of superconductivity, but clearly is due to experimental artifacts or properties of the background, strongly suggests that the similar observed behavior shown for the sample after heating in the middle panels in fig. SI1, interpreted by the authors as due to superconductivity, is equally caused by the same experimental artifacts or properties of the 

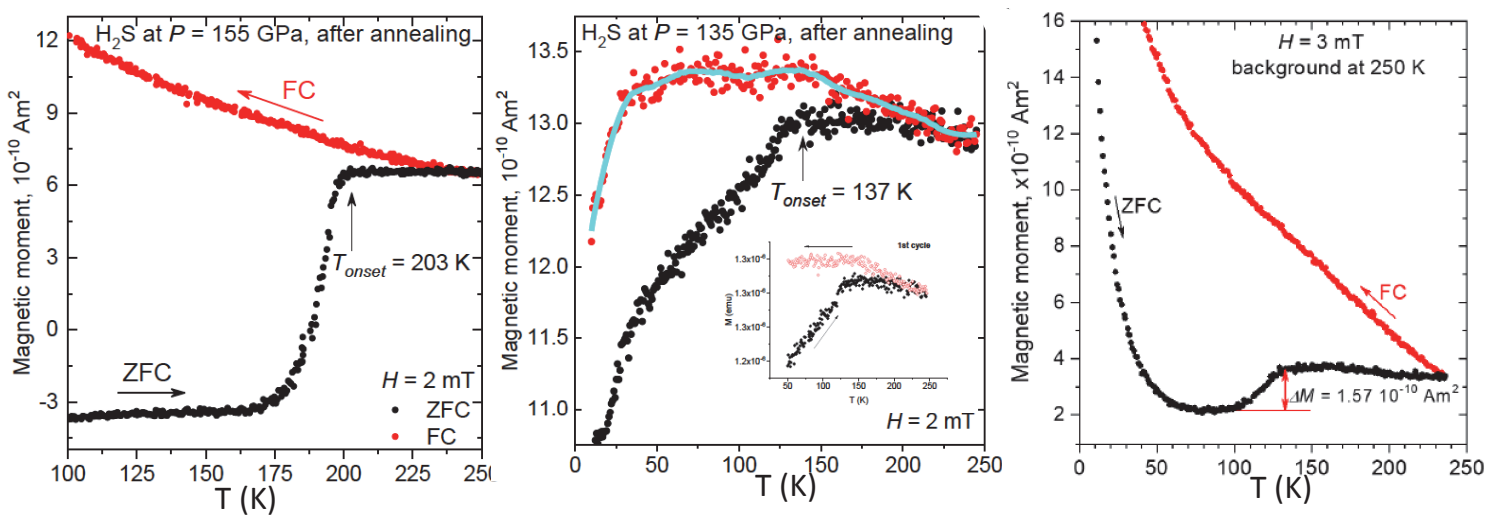

FIG. 5: Magnetic moment versus temperature for sulfur hydride under field cooled (red points) and zero field cooled (black points) conditions for three different runs performed in 2015, reported in ref. [3] in 2015 (left panel) and in ref. [2], fig. 5 (b) (center panel) and fig. 6 (a) (right panel).

background.

\section{EREMETS ET AL. 2022: 'EXPERIMENTAL EVIDENCE AND DETAILS'}

In reference [2], Eremets and coworkers describe in greater detail the experiments that in their view reveal high temperature superconductivity in hydrides under high pressure. Here we address several of the points made in that paper posted in January 2022, more than 7 years after the announced discovery of high temperature superconductivity in sulfur hydride [3, 4].

In the introduction of [2], the authors argue that nuclear resonant scattering experiments [31], infrared spectroscopy [33] and ac susceptibility measurements [34] have supported superconductivity. We have provided elsewhere detailed arguments for why those experiments were flawed [14, 16, 18, 20]. The authors of [2] ignore the issues that we raised in those references. In particular, they state in connection with ref. [31] that "it appears that the nuclear resonance scattering is a new, non-trivial, and sophisticated technique to detect superconductivity. Hopefully it will find further use to study novel near room-temperature superconductors in difficult conditions, such as ultra-high pressure, and, perhaps in compounds exhibiting superconductivity even above room temperature." We point out that it is peculiar that in the ensuing 6 years since ref [31] was published not a single experiment using this technique was reported, neither for a hydride nor for any other suspected or confirmed superconductor. Nor was that technique used to detect the Meissner effect of any superconductor ever before the experiment reported in [31].

Fig. 5 shows measurements of the magnetic moment of sulfur hydride versus temperature by the Eremets group around the year 2015. The left panel was published in the 2015 paper [3], the center and right panel were unpub- lished until 2022 [2]. The left panel suggests a transition to superconductivity, and the magnetic moment changes from positive to negative as expected for a superconducting transition. Instead, in the center and right panels the magnetic moment does not change sign. Additionally, the magnitude of the drop in magnetic moment is a factor of 10 smaller on the right panel compared to the left panel. For the center panel, the drop in magnetic moment occurs over a temperature interval of about $100 \mathrm{~K}$, versus a temperature interval of about $20-30 \mathrm{~K}$ for the left and right panels. Over that interval, the drop in magnetic moment in the center panel is a factor of 5 smaller than in the left panel.

Why are these three panels so different from each other? The magnitude of the magnetic moment drop should only depend on sample dimensions, not temperature or pressure, and the sample dimensions for the three cases were presumably similar. The width of the transition should be similar in different experiments but it is not.

The fact that there are all these differences between the three panels suggests that the behavior observed is not due to superconductivity. It is interesting that the authors chose to show only the left panel in their published work 7 years ago [3], which is presumably related to the fact that it shows something closer to what is expected for a superconducting transition than the other two panels.

The strongest evidence for magnetic field expulsion under field cooling, i.e. Meissner effect proper, is provided by the authors of [2] in their Fig. 5h. In Fig. 6 we reproduce their Fig. 5h in the upper panel, and in the lower panel we show the same figure with the blue line drawn by hand removed. We argue that "The subtle step(s) observed on FC curves" that according to the authors of [2] is indicated by the blue line in the upper panel is no longer apparent in the lower panel when the blue line and the arrow are removed. 


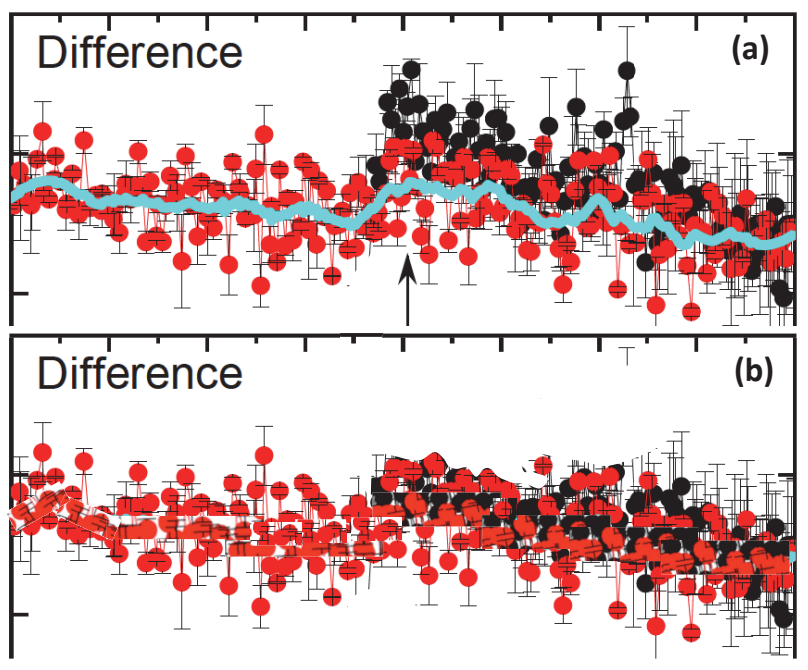

FIG. 6: Upper panel: alleged signature of the Meissner effect upon field cooling indicated by the blue line, from ref. [2] Fig. 5h. Lower panel: same picture with the blue line removed, i.e. we simply replaced the blue line by less distracting red points and white background that now hide the blue line. We also removed some distracting black points. All remaining black points should be ignored - they correspond to zero field cooled measurements. There is no apparent transition as a function of temperature indicated by the red points in the lower panel.

Regarding sample dimensions, Eremets et al. in 2022 [2] start in the introduction by informing readers that "the typical size of samples residing in a diamond anvil cell $(D A C)$ is necessarlly small - of the order of $\sim 50 \mu \mathrm{m}$ in lateral dimensions". The word "necessarily" in this context is a fallacy.

The maximum pressure that can be achieved in a diamond anvil cell is a function of the culet dimensions. This is discussed for example in ref. [35] and references therein. In their 2015 paper [3], Eremets et al. reportedly used "a culet of $40-80 \mu \mathrm{m}$ " and reported measurements in the range 100 - $200 \mathrm{GPa}$. In their 2021 paper, Eremets et al. reported using culet sizes of $\sim 75$ and $\sim 90 \mu \mathrm{m}$ and pressures up to $167 \mathrm{GPa}$ were applied.

While for the higher pressures the smaller size culet may be necessary, superconductivity was reportedly detected in ref. [3] at around $120 \mathrm{GPa}$. Such a pressure can be easily achieved with a $200 \mu \mathrm{m}$ culet and possibly even larger [35]. Such culets should allow for sample diameters much larger than the $\sim 50 \mu \mathrm{m}$ that Eremets et al. tells us in 2022 are the sizes of the "tiny samples" that necessarily have to be used. If the samples were not so "tiny" but instead closer to $200 \mu \mathrm{m}$ in diameter or even larger and correspondingly thicker, it should be much easier to unambiguously demonstrate a magnetic signature of superconductivity if it existed.

Regarding other measurements, particularly resistance, the 2022 paper by Eremets et al. [2] states: " $H_{3} S$ was independently synthesized and superconductivity was fully confirmed [12-16]". We remark that:

(i) Ref. [12] [36] (2018) is coauthored by Eremets, so it hardly qualifies as 'independent'. The single resistance curve shown there showing a drop starting at temperature above $150 \mathrm{~K}$ has a width of more than $70 \mathrm{~K}$, significantly larger than the resistive transitions shown in ref. [3] of width $10 \mathrm{~K}$ or less.

(ii) Ref. [13] [37] (2020) is not coauthored by Eremets but the single experimental graph indicating superconductivity is the same resistance measurement reported in ref. [12] [36].

(iii) Ref. [15] [39] (2019) is not coauthored by Eremets, it reports a single experimental curve of resistance versus temperature showing a $15 \%$ drop in resistance at $200 K$, and the same curve with "subtraction of residual resistance", i.e. subtracting $85 \%$ of the resistance, showing that it now drops (not surprisingly) to zero.

(iv) Ref. [14] [38] (2019) shows the same experimental curve (with residual resistance subtracted) as ref. [15] [39].

(v) Ref. [16] [34] are the ac susceptibility measurements that ref. [20] showed to be flawed.

We argue that the above calls the statement of "fully confirmed" made in ref. [2] based on its references [12-16] into question.

Finally, we would like to comment on the implication of the following paragraph of Eremets et al. 2022 paper [2] reproduced below in full:

"For a long time we did not clearly appreciate the role played (by) the quality and integrity of the samples. Even when the sample has a large enough size and clearly shows superconductivity in electrical transport measurements, the magnetic susceptibility signal can turn out to be elusive or below the sensitivity of the SQUID magnetometer. The reason for that can be the granular or non-uniform distribution of the superconducting phase in samples. The electrical current finds a continuous path through superconducting grains and metallic grain boundaries in the transport measurements whereas much smaller thin superconducting grains have a relatively small superconducting volume leading to a smaller signal due to the demagnetization factor."

The authors explicitly acknowledge that current circulating through "metallic grain boundaries" providing "a continuous path" can be interpreted as showing superconductivity in their experiments. If their superconducting grains have a "relatively small superconducting volume" compared to the total volume it implies that a significant part of the path through which the current circulates is metallic and not superconducting. If the current necessarily has to go through metallic grain boundaries, which necessarily have non-zero resistance, the material cannot sustain a persistent current and cannot possess macroscopic phase coherence. We argue that a material that cannot exhibit these defining features of superconductivity cannot be called a superconductor, irrespective of how small its resistance is or how much its resistance depends on the ionic mass. 


\section{A LITMUS TEST FOR HOT HYDRIDE SUPERCONDUCTIVITY}

We have proposed in ref. [16] that detection of trapped flux would provide convincing evidence for the existence of persistent currents in these materials. It would appear that to do this test would not be more difficult than to perform the measurements reported in refs. [1] and [3].

To rationalize the complete absence of flux expulsion upon field cooling seen in the left panel of fig. 1, one could hypothesize that there is a large concentration of defects that trap the magnetic field and prevent it from being expelled, even at the large cost of condensation energy implied by the very large $H_{c}$. Let us entertain that possibility. If after the field cooling process shown on the left panel of Fig. 1, for applied magnetic field smaller than $H_{c 1}$, the applied field is turned off, those defects should prevent the interior magnetic field from decaying and a remnant magnetization should be detectable for several hours, days or months thereafter. As shown in refs. [40, 41], the remnant magnetic moment should be given approximately by the difference in the FC and ZFC moments for the same magnetic field.

So far there have not been experimental reports that trapped flux, persisting for a long time, has been detected in hydrides. This is in contrast to what happened with the cuprates, where from the very beginning it was seen that samples that did not strongly expel magnetic fields trapped magnetic fields and this was considered independent evidence for superconductivity [42]. The current situation for hydrides is depicted in Fig. 7. Standard superconductors, conventional and unconventional, are described by the extreme behaviors shown in the first and second row of Fig. 7, as well as behavior intermediate between them, i.e. part of the flux is expelled upon cooling and the part that is not expelled remains trapped after the external field is removed. Thus far hydride superconductors, and non-superconductors, are described by the third row: none of the flux is expelled upon field cooling, and none is trapped when the external field is removed.

If such a trapped flux is detected for a hydride sample in the future, and it is not detected when the same process is performed for the precursor sample before heating, it will provide convincing evidence that persistent currents flow in the material in the absence of applied magnetic field. Then the unlikely possibility that these materials are 'nonstandard superconductors' [13, 14] will have to be seriously considered. Conversely, if no field trapping is observed, it will provide strong confirmation of our arguments that indicate that these systems are not superconducting.

Similarly we have suggested [16] that in a nuclear resonant scattering experiment (NRS) [31] that claimed to show flux exclusion from sulfur hydride from the absence of quantum beats in the NRS spectra under an applied magnetic field, it would be straightforward to verify the presence of trapped field by showing that quantum beats

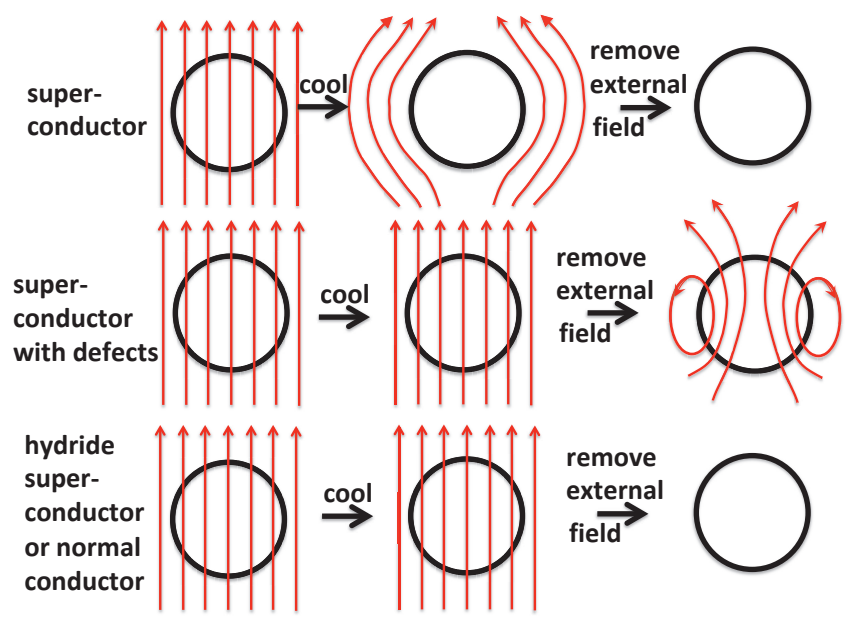

FIG. 7: First two rows: expected behavior of standard superconductors. The first row shows complete flux expulsion, as occurs in clean type I superconductors or type II superconductors with weak pinning centers. The second row shows small or zero Meissner fraction, as occurs in superconductors with many defects/strong pinning centers that trap magnetic field. When the external field is removed, the trapped field remains. Of course intermediate behavior between the first and second row is possible and in fact most common, namely some of the flux is expelled and some is trapped when the external field is removed. The third row shows the behavior of normal metals and of hydride superconductors (as far as we know to date): they neither expel magnetic field nor trap magnetic field.

are present after field cooling and removal of the external field.

\section{SUMMARY AND CONCLUSIONS}

The magnetic measurements reported in ref. [1] were intended to establish that hydrides under pressure are indeed high temperature superconductors. The paper claims to present unambiguous (three times) and definitive (once) evidence for high temperature superconductivity in sulfur hydride and lanthanum hydride under pressure. Ref. [2] claims to have presented "very solid evidence for high-temperature superconductivity in hydrogen-rich compounds under high pressures". In this paper we argued that instead these recent experimental papers $[1,2]$ not only have not established that these hydrides are superconductors but rather they have increased the likelihood that they are not. They give a snapshot of where the field is today, more than 7 years after the reported discovery of this new class of superconducting materials. Compare this snapshot with a snapshot of where the field of cuprate superconductivity was in 1991 [43], 5 years after the discovery of that class of materials. The difference is stark.

Let us summarize our conclusions.

(1) The measurements show that if the materials are 
superconductors, they have critical current values that are one to three orders of magnitude larger than those of all standard superconductors.

(2) Their lower critical field and thermodynamic critical field are between one and two orders of magnitude larger than for any standard superconductor.

(3) Their density of states is between one and two orders of magnitude larger than what is expected for a material with such composition.

(4) Their London penetration depth is at least a factor of 2 smaller than that of any known superconductor, including very pure elements.

Conclusions (1) to (4) are based on our assumption, consistent with the authors' claims, that the lower critical field in these materials, if they are superconductors, is of order $0.5 \mathrm{~T}$ or larger. If instead the critical field was one or two orders of magnitude smaller, these conclusions would be invalidated. We do not believe that this is plausible, given the magnetic evidence discussed.

(5) They show absolutely no evidence for magnetic field expulsion in the presence of a magnetic field (figs. 2c and 2d in ref. [1]). This is completely unprecedented for weakly type II superconductors with such small values of the London penetration depth that imply very high values for the superfluid densities.

(6) The sample preparation process indicates that the resulting samples are not single crystals and have weak links and disorder, making even more implausible the claimed large values of lower critical field and critical current, and low value of the London penetration depth.

(7) The measured magnetic moment versus temperature under zero field cooling conditions, except for very small magnetic field values, shows monotonic behavior of approximately constant slope (Fig. 3) with absolutely no signature of a superconducting transition at the expected values of the critical temperature.

(8) The measured magnetic moment interpreted as due to superconductivity is five times smaller than the magnetic moment interpreted as due to superconductivity in the 2015 paper [3] for a sample of similar size.

(9) The measured magnetic moment versus temperature in the presence of a small magnetic field for precursor nonsuperconducting samples shows similar behavior to the behavior allegedly signaling superconductivity in the alleged superconducting samples.

(10) Detection of trapped flux [16] that persists for long periods after field cooling and removal of the external field has not been reported so far. If it is found, it would show that the materials can carry persistent currents, hence it is a superconductor. This is assuming that contributions to magnetic remanence from magnetic sources can be ruled out, e.g. by comparison with the behavior of untreated samples not expected to be superconducting. If trapped flux is looked for and not found, its absence would confirm that the materials are not superconductors.

It should also be pointed out that ref. [1] misleadingly claims that "the Meissner effect" was demonstrated, even in the title. The fact is, the Meissner effect is magnetic field expulsion, not magnetic field exclusion. Magnetic field exclusion was known to researchers in 1911, magnetic field expulsion was only discovered in 1933. Ref. [1] showed zero evidence for magnetic field expulsion. Similarly ref. [31] claimed to report "direct observation of the expulsion of the magnetic field" from sulfur hydride, when in fact no field cooling was even performed.

The experiments reported by Minkov et al. [1] and Eremets et al. [2] are performed under very difficult experimental conditions, at very large pressures and with very small samples, and a variety of effects unrelated to the physics of the sample but instead due to properties of the background or the experimental apparatus could come into play. Under such challenging conditions we suggest that it is very important to guard against confirmation bias, the tendency to pay undue attention to observed features that confirm prior beliefs and ignore others that don't. We don't know what could be the origin of the features found in the experiments that are attributed to be superconductivity by Eremets et al., but have argued in this paper that in our opinion they are inconsistent with superconductivity.

In other recent papers we have analyzed various other reported evidence for superconductivity in pressurized hydrides and concluded that every experiment was flawed, namely:

(1) ac magnetic susceptibility reported to show a superconducting transition in sulfur hydride [34] was shown to be due to an experimental artifact [20].

(2) Optical reflectance measurements that reportedly showed that sulfur hydride is a superconductor [33] were shown to be flawed [18].

(3) The reported observation of a Meissner effect in sulfur hydride using nuclear resonant scattering [31] was shown to be flawed [14].

(4) Magnetic susceptibility measurements for a room temperature superconducting hydride [44] were shown to be flawed [21-23].

(5) Magnetic susceptibility measurements reported for lanthanum hydride showed weak and very broad peaks [45], inconsistent with the width of the presumed transition shown in resistivity measurements of that material $[15,46,47]$.

(6) Resistance measurements for a room temperature superconducting hydride [44] and several other hydrides [3, 47-49] were shown to be anomalously sharp, and/or with an unchanging width in the presence of an applied magnetic field, indicating that the drops in resistance were not due to superconductivity $[12,13,17]$.

In addition, an alternative theory of superconductivity predicts that high temperature superconductivity in this class of materials should not exist [50, 51].

The field of high temperature superconductivity in hydrides was launched in 2015 by the publication of ref. [3] by Eremets and coworkers. Now, the same author and coworkers present evidence $[1,2]$ that (a) invalidates the evidence for superconductivity presented in the 2015 pa- 
per and (b) is in itself flawed, as discussed in this paper. From this, together with our analysis of the totality of magnetic evidence and other experimental evidence for superconductivity in hydrides discussed in our earlier papers, we argue that the most likely conclusion is that hydrides under pressure are not high temperature superconductors. Whatever the origin of their anomalous behavior is, whether intrinsic or due to experimental artifacts or both, in our opinion it is not due to superconductivity.

\section{Acknowledgments}

We are grateful to D. Semenok for calling ref. [1] to our attention, to V. S. Minkov, S. L. Bud'ko, R. Pro- zorov and M. I. Eremets for clarifying correspondence on their papers [1, 2], and to V. Struzhkin for discussions. JEH would like to thank S. Shylin for extensive discussions and sharing of information on refs. [3] and [2]. FM was supported in part by the Natural Sciences and Engineering Research Council of Canada (NSERC), and by an MIF from the Province of Alberta.
[1] V. S. Minkov et al, "The Meissner effect in hightemperature hydrogen-rich superconductors under high pressure", DOI:10.21203/rs.3.rs-936317/v1 (2021).

[2] M. I. Eremets et al, "High-temperature superconductivity in hydrides: experimental evidence and details", arXiv:2201.05137 (2022).

[3] A.P. Drozdov, M.I. Eremets, I. A.Troyan, V. Ksenofontov and S. I. Shylin, 'Conventional superconductivity at 203 kelvin at high pressures in the sulfur hydride system', Nature 525, 73-76 (2015).

[4] A.P. Drozdov, M.I. Eremets and I. A.Troyan, "Conventional superconductivity at $190 \mathrm{~K}$ at high pressures", arXiv:1412.0460 (2014).

[5] D. V. Semenok et al, "On Distribution of Superconductivity in Metal Hydrides", Current Opinion in Solid State and Materials Science 24, 100808 (2020) and references therein.

[6] G. Gao et al, "Superconducting Binary Hydrides: Theoretical Predictions and Experimental Progresses", Materials Today Physics 100546 (2021).

[7] X. Zhang et al, "Pressure-induced hydride superconductors above 200 K", Matter and Radiation at Extremes 6, 068201 (2021).

[8] F. Marsiglio and J. Carbotte, in "Superconductivity", edited by K. Bennemann and J. Ketterson, Vol. 1, p. 73 (Springer, Berlin, 2008).

[9] C. J. Pickard, I. Errea and M. I. Eremets, "Superconducting Hydrides Under Pressure", Ann. Rev. Cond. Matt. Phys. 11, 57 (2020) and references therein.

[10] J. A. Flores-Livas et al., "A perspective on conventional high-temperature superconductors at high pressure: Methods and materials", Physics Reports 856, 1 (2020).

[11] L. Boeri et al, "The 2021 Room-Temperature Superconductivity Roadmap", J. Phys. Cond. Matt., to be published.

[12] J. E. Hirsch and F. Marsiglio, "Absence of high temperature superconductivity in hydrides under pressure", arXiv:2010.10307 (2020).

[13] J. E. Hirsch and F. Marsiglio, "Nonstandard superconductivity or no superconductivity in hydrides under high pressure", Phys. Rev. B 103, 134505 (2021).
[14] J. E. Hirsch and F. Marsiglio, "Meissner effect in nonstandard superconductors", Physica C 587, 1353896 (2021).

[15] J. E. Hirsch and F. Marsiglio, "Absence of magnetic evidence for superconductivity in hydrides under high pressure", Physica C 584, 1353866 (2021).

[16] J. E. Hirsch and F. Marsiglio, "Flux trapping in superconducting hydrides under high pressure", Physica C 589, 1353916 (2021).

[17] J. E. Hirsch and F. Marsiglio, "Unusual width of the superconducting transition in a hydride", Nature 596, E9 (2021).

[18] J. E. Hirsch and F. Marsiglio, "Absence of evidence of superconductivity in sulfur hydride in optical reflectance experiments", arXiv:2109.10878 (2021).

[19] J. E. Hirsch, "About the Pressure-Induced Superconducting State of Europium Metal at Low Temperatures", Physica C 583, 1353805 (2021).

[20] J. E. Hirsch, "Faulty evidence for superconductivity in ac magnetic susceptibility of sulfur hydride under pressure", arXiv:2109.08517 (2021).

[21] J. E. Hirsch, "On the ac magnetic susceptibility of a room temperature superconductor: anatomy of a probable scientific fraud", Physica C doi.org/10.1016/j.physc.2021.1353964 (2021), arXiv:2110.12854v1 (2021).

[22] J. E. Hirsch, "Disconnect between Published AC Magnetic Susceptibility of a Room Temperature Superconductor and Measured Raw Data", Preprints 2021, 2021120115.

[23] J. E. Hirsch, "On the room temperature superconductivity of carbonaceous sulfur hydride", Europhys. Lett. doi.org/10.1209/0295-5075/ac50c9 (2022).

[24] M. Dogan and M. L. Cohen, "Anomalous behavior in high-pressure carbonaceous sulfur hydride", Physica C 583, 1353851 (2021).

[25] E. F. Talantsev, "The electron-phonon coupling constant, Fermi temperature and unconventional superconductivity in the carbonaceous sulfur hydride $190 \mathrm{~K}$ superconductor", Supercond. Sci. Technol. 34, 034001 (2021).

[26] L. Mazov, "Comment on "Conventional superconductivity at 203 kelvin at high pressures in the sulfur hydride 
system ", arXiv:1510.00123 (2015); "Interplay between magnetism and superconductivity in metallic hydrogen and hydrides at high pressure", EPJ Web of Conferences 185, 08003 (2018).

[27] T. Wang et al, "Absence of conventional roomtemperature superconductivity at high pressure in carbon-doped $H_{3} S^{\prime \prime}$, Phys. Rev. B 104, 064510 (2021).

[28] M. Gubler et al, "Missing theoretical evidence for conventional room temperature superconductivity in low enthalpy structures of carbonaceous sulfur hydrides", arXiv:2109.10019 (2021).

[29] N.M. Nusran et al, "Spatially-resolved study of the Meissner effect in superconductors using NV-centersin-diamond optical magnetometry", New J. Phys. 20, 043010 (2018).

[30] A. S. Sefat et al, "Superconductivity at $22 \mathrm{~K}$ in Co-Doped $\mathrm{BaFe}_{2} \mathrm{As}_{2}$ Crystals", Phys. Rev. Lett. 101, 117004 (2008).

[31] I. Troyan et al, "Observation of superconductivity in hydrogen sulfide from nuclear resonant scattering", Science 351, 1303 (2016).

[32] E. Talantsev, W. P. Crump and J. L. Tallon, "Thermodynamic Parameters of Single- or Multi-Band Superconductors Derived from Self-Field Critical Currents", Ann. der Physik 529, 1700197 (2017).

[33] F. Capitani et al, "Spectroscopic evidence of a new energy scale for superconductivity in $H_{3} S$ ", Nat. Phys. 13, 859 (2017).

[34] X. Huang et al, "High-temperature superconductivity in sulfur hydride evidenced by alternating-current magnetic susceptibility", Nat. Sci. Rev. 6, 713 (2019).

[35] E.F. O'Bannon III et al, "Contributed Review: Culet diameter and the achievable pressure of a diamond anvil cell: Implications for the upper pressure limit of a diamond anvil cell", Review of Scientific Instruments 89, 111501 (2018).

[36] K. Shimizu et al, "Superconductivity and structural studies of highly compressed hydrogen sulfide", Physica C 552, 27 (2018).

[37] K. Shimizu, "Investigation of Superconductivity in Hydrogen-rich Systems", Journal of the Physical Society of Japan 89, 051005 (2020).

[38] R. Akashi, "Evidence of Ideal Superconducting Sulfur
Superhydride in a Pressure Cell", JPSJ News Comments 16, 18 (2019).

[39] H. Nakao et al, "Superconductivity of Pure $H_{3} S$ Synthesized from Elemental Sulfur and Hydrogen", J. Phys. Soc. Jpn. 88, 123701 (2019).

[40] A. P. Malozemoff et al, "Remanent moment of hightemperature superconductors: Implications for fluxpinning and glassy models", Phys. Rev. B 38, 6490 (1988).

[41] A. K. Grover et al, "Thermomagnetic history effects in niobium and its implication for $H_{c 1}$ in high $T_{c}$ superconductors", Pramana 33, 297 (1989).

[42] K. A. Müller, M. Takashige and and J. G. Bednorz, "Flux Trapping and Superconductive Glass State in $\mathrm{La}_{2} \mathrm{CuO}_{4-y}$ : Ba", Phys. Rev. Lett. 58, 1143 (1987).

[43] Proceedings of the International Conference on Materials and Mechanisms of Superconductivity - High Temperature Superconductors, Kanazawa, Japan, July 22 to 26 July, 1991, Physica C Volumes 185-189, Part I, Part II, Part III, Part IV (1991).

[44] E. Snider et al., "Room-temperature superconductivity in a carbonaceous sulfur hydride", Nature 586, 373 (2020).

[45] V. Struzhkin et al, "Superconductivity in La and Y hydrides: Remaining questions to experiment and theory", Matter and Radiation at Extremes 5, 028201 (2020).

[46] M. Somayazulu et al., "Evidence for superconductivity above $260 \mathrm{~K}$ in lanthanum superhydride at megabar pressures", Phys. Rev. Lett. 122, 027001 (2019).

[47] A. P. Drozdov et al, "Superconductivity at $250 \mathrm{~K}$ in lanthanum hydride under high pressures", Nature 569, 528 (2019).

[48] D. V. Semenok et al, "Superconductivity at $253 \mathrm{~K}$ in lanthanum-yttrium ternary hydrides", Materials Today 48, 18 (2021).

[49] P. Kong et al, "Superconductivity up to $243 \mathrm{~K}$ in the yttrium-hydrogen system under high pressure", Nature Comm. 12, 5075 (2021).

[50] See https://jorge.physics.ucsd.edu/hole.html for a list of references.

[51] J. E. Hirsch, "Hole superconductivity xOr hot hydride superconductivity", J. Appl. Phys. 130, 181102 (2021). 